\title{
Boas práticas de enfermagem relacionadas ao uso de sonda enteral
}

\author{
Good nursing practices related to the use of enteral probe \\ Buenas prácticas de enfermería relacionadas con el uso de la sonda enteral
}

\author{
Aline Sandra Gomes Corrêa \\ ORCID: https://orcid.org/0000-0001-9055-854X \\ Universidade do Estado do Rio de Janeiro, Brasil \\ E-mail: alinesg.correa@gmail.com \\ Célia Regina Gonçalves Ferreira \\ ORCID: https://orcid.org/0000-0003-4121-6900 \\ Universidade do Estado do Rio de Janeiro, Brasil \\ E-mail: celia__regina25@hotmail.com \\ Glauver Alves Moreira \\ ORCID: https://orcid.org/0000-0003-1016-9056 \\ Universidade do Estado do Rio de Janeiro, Brasil \\ E-mail: glauvermoreira@yahoo.com \\ Herley de Oliveira Diniz Junqueira \\ ORCID: https://orcid.org/0000-0001-9223-3220 \\ Universidade do Estado do Rio de Janeiro, Brasil \\ E-mail: herley.diniz@gmail.com \\ Luana Ferreira de Almeida \\ ORCID: https://orcid.org/0000-0001-8433-4160 \\ Universidade do Estado do Rio de Janeiro, Brasil \\ E-mail: luana.almeida3011@gmail.com \\ Sandra Regina Maciqueira Pereira \\ ORCID: https://orcid.org/0000-0002-0550-2494 \\ Universidade do Estado do Rio de Janeiro, Brasil \\ E-mail: sandregina@gmail.com \\ Vanessa Galdino de Paula \\ ORCID: https://orcid.org/0000-0002-7147-5981 \\ Universidade do Estado do Rio de Janeiro, Brasil \\ E-mail: vanegalpa@gmail.com
}

\begin{abstract}
Resumo
Objetivo: Identificar as boas práticas de enfermagem, relacionadas ao uso de sonda enteral. Metodologia: Revisão integrativa, a partir de publicações indexadas nas bases de dados: Literatura Latino-Americana e do Caribe (LILACS), Base de Dados de Enfermagem (BDENF), Cumulative Index to Nursing and Allied Health Literature (CINAHL), $\mathrm{O}$ portal PubMed e a biblioteca digital Scientific Electronic Library Online (SCIELO). Foram incluídos na busca estudos disponíveis em texto completo, publicados entre janeiro de 2014 a abril de 2019, nos idiomas português, inglês e espanhol, relacionados a pacientes acima de 18 anos. Excluídos aqueles com duplicidade e que não correspondiam a temática. Na consulta às bases de dados, foram utilizados os Descritores em Ciências da Saúde (DeCS): “Alimentação por tubo", "Cuidados de enfermagem"; "Intubação Gastrointestinal", associados através do conector boleano "and". Posteriormente, os artigos foram categorizados quanto ao título, autores, periódico, ano de publicação, país e tipo de estudo. Resultados: Foram selecionados 15 artigos que atendiam a todos os critérios estabelecidos, resultando em quatro categorias temáticas: "Uso de protocolos relacionados ao uso de sonda enteral"; "Verificação do posicionamento da sonda enteral"; "Conhecimento sobre terapia nutricional enteral" e "Prevenção de eventos adversos". Conclusão: Observou-se que as boas práticas relacionadas ao uso de sonda enteral vão além da correta instalação da própria, abrangendo também as orientações à família e equipes e principalmente cumprimento dos protocolos institucionais.
\end{abstract}

Palavras-chave: Alimentação por tubo; Cuidados de enfermagem; Intubação gastrointestinal.

\section{Abstract}

Objective: To identify good nursing practices related to the use of enteral probes. Methodology: Integrative review, from publications indexed in the databases: Latin American and Caribbean Literature (LILACS), Nursing Database (BDENF), Cumulative Index to Nursing and Allied Health Literature (CINAHL), pubmed portal and digital library Scientific Electronic Library Online (SCIELO). The search included studies available in full text, published between January 2014 and April 2019, in the Portuguese, English and Spanish languages related to patients over 18 years of age. Excluded those with duplicity and who did not correspond to the theme. In the consultation of the databases, the 
Descriptors in Health Sciences (DeCS) were used: "Feeding by tube", "Nursing care"; "Gastrointestinal intubation", associated through the bolean connector "and". Subsequently, the articles were categorized according to title, authors, journal, year of publication, country and type of study. Results: We selected 15 articles that met all established criteria, resulting in four thematic categories: "Use of protocols related to the use of enteral probe"; "Verification of enteral probe positioning"; "Knowledge about enteral nutritional therapy" and "Prevention of adverse events". Conclusion: It was observed that good practices related to the use of enteral probego beyond the correct installation of its own, also covering the guidelines to the family and teams and mainly compliance with institutional protocols.

Keywords: Tube feeding; Nursing care; Gastrointestinal intubation.

\section{Resumen}

Objetivo: Identificar buenas prácticas de enfermería relacionadas con el uso de sondas enterales. Metodología: Revisión integrativa, a partir de publicaciones indexadas en las bases de datos: Literatura Latinoamericana y del Caribe (LILACS), Base de Datos de Enfermería (BDENF), Índice Acumulativo de Literatura de Enfermería y Salud Aliada (CINAHL), portal pubmed y biblioteca digital Biblioteca Electrónica Científica en Línea (SCIELO). La búsqueda incluyó estudios disponibles en texto completo, publicados entre enero de 2014 y abril de 2019, en los idiomas portugués, inglés y español relacionados con pacientes mayores de 18 años. Excluidos aquellos con duplicidad y que no correspondían al tema. En la consulta de las bases de datos, se utilizaron los Descriptores en Ciencias de la Salud (DeCS): "Alimentación por tubo", "Cuidado de enfermería"; "Intubación gastrointestinal",asociada através del conector bolean " $y$ ". Posteriormente, los artículos fueron categorizados según el título, autores, revistas, año de publicación, país y tipo de estudio. Resultados: Seleccionamos 15 artículos que cumplían todos los criterios establecidos, lo que dio lugar a cuatro categorías temáticas: "Uso de protocolos relacionados con el uso de sonda enteral"; "Verificación del posicionamiento de la sonda enteral"; "Conocimiento sobre la terapia nutricional enteral" y "Prevención de eventos adversos". Conclusión: Se observó que las buenas prácticas relacionadas con el uso de probego enteral más allá de la correcta instalación de los suyos, abarcando también las directrices a la familia y los equipos y principalmente el cumplimiento de los protocolos institucionales.

Palabras clave: Alimentación de tubos; Cuidado de enfermería; Intubación gastrointestinal.

\section{Introdução}

A nutrição enteral (NE) é comumente utilizada em pacientes internados em unidade de terapia intensiva. Isso se justifica, pois muitas vezes estes possuem uma demanda metabólica acima da média, em virtude de diversas patologias que podem levá-los a um intenso catabolismo lipídico e proteico, principalmente na fase aguda da doença (Viana \& Torre, 2016; Nettina, 2014).

Nesse sentido, a instalação e manutenção de sonda enteral (SE) busca garantir um suporte nutricional adequado, e consequentemente o reestabelecimento das funções orgânicas do paciente crítico. Trata-se de uma medida terapêutica que visa diminuir a probabilidade de alterações no sistema imunológico, tegumentar, entre outros sistemas, que a perda nutricional pode afetar. Mesmo diante da necessidade e importância do uso de SE em pacientes críticos, problemas relacionados ao seu uso podem ocorrer (Pereira et al, 2018; Colaço \& Nascimento, 2014).

Eventos adversos relacionados às SE correspondem a obstrução do tubo de alimentação, saída inadvertida do cateter e à alteração do volume da dieta, que se pretende infundir podendo ser ofertado menos que o estimado. Tais eventos podem ocorrer por falta de lavagem com água filtrada antes e após a administração de medicamentos e dietas, precipitação da dieta, dobras da sonda e retirada pelo paciente (Cervo et al, 2014).

Além disso, muitas vezes, o volume ofertado menor que o recomendado, ocorre devido a interrupções da nutrição enteral durante procedimentos. A diferença significativa entre os conteúdos prescritos e administrados, pode levar a complicações gastrointestinais (emêse, diarreia ou constipação), contribuindo diretamente para a ocorrência de eventos adversos e consequentemente para um possível comprometimento do estado nutricional do paciente (De Souza, 2018).

Diante dos problemas relacionados ao uso de SE e da necessidade desta, para o atendimento das carências metabólicas do paciente crítico, é fundamental que os enfermeiros assumam um papel ativo na prevenção de incidentes e eventos adversos referentes a esse dispositivo (O'Leary-Kelley \& Bawel-Brinkley, 2017). 
Desta forma, surgiu a seguinte questão norteadora: Quais as boas práticas de enfermagem relacionadas ao uso de SE, descritas na literatura? Para responder a pergunta, o estudo teve como objetivo: identificar as boas práticas de enfermagem, relacionadas ao uso de SE.

\section{Metodologia}

Trata-se de uma revisão integrativa, que tem por finalidade permitir a análise de subsídios na literatura de forma ampla e sistemática, além de divulgar dados científicos produzidos por outros autores (Ramalho et al, 2016). A revisão integrativa consiste no cumprimento das etapas: identificação do tema e seleção da questão de pesquisa; estabelecimento dos critérios de elegibilidade; identificação dos estudos nas bases científicas; avaliação dos estudos selecionados e análise crítica; categorização dos estudos; avaliação e interpretação dos resultados e apresentação dos dados na estrutura da revisão integrativa (Botelho, Cunha \& Macedo, 2011). Portanto, elaborou-se a seguinte questão norteadora: Quais as boas práticas de enfermagem, relacionadas ao uso de SE em pacientes críticos adultos?

Para respondê-la, foram consultadas as bases de dados: Literatura Latino-Americana e do Caribe (LILACS), Base de Dados de Enfermagem (BDENF), Cumulative Index to Nursing and Allied Health Literature (CINAHL, o portal PubMed e a biblioteca digital Scientific Electronic Library Online (SCIELO). Foram incluídos na busca estudos disponíveis em texto completo, publicados entre janeiro de 2014 a abril de 2019, nos idiomas português, inglês e espanhol, relacionados a pacientes acima de 18 anos. Excluídos aqueles com duplicidade e que não correspondiam a temática.

$\mathrm{Na}$ consulta às bases de dados, foram utilizados os Descritores em Ciências da Saúde (DeCS): "Alimentação por tubo", "Cuidados de enfermagem"; "Intubação Gastrointestinal". Posteriormente, os artigos foram categorizados quanto ao título, autores, periódico, ano de publicação, país e tipo de estudo. Com a associação dos descritores através do conector boleano "and", foram identificados 4.957 artigos nas bases consultadas, sendo 15 artigos estabelecidos como corpo de análise, de acordo com a Figura 1. 
Figura 1 - Fluxograma sobre a seleção de artigos após critérios de inclusão e exclusão. Rio de Janeiro, RJ, Brasil, 2019.

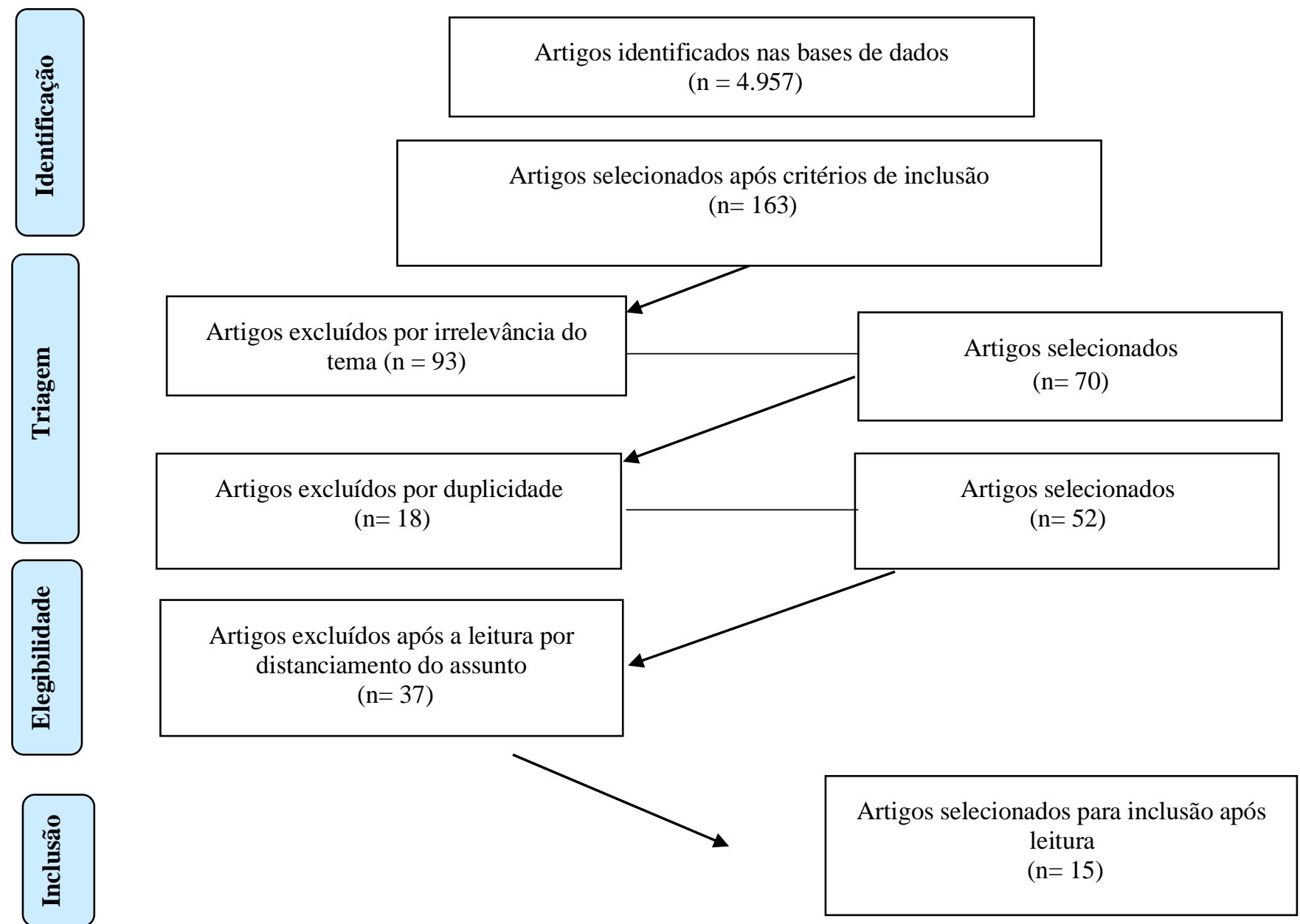

Fonte: Dados da pesquisa (2019).

\section{Resultados e Discussão}

Entre os 15 artigos selecionados, pode-se verificar que 4 (26,6\%) foram publicados nos anos de 2015 e 2014 , respectivamente. Dois (13,3\%) nos anos de 2016, 2018, 2019, respectivamente; e $1(6,6 \%)$ artigo em 2017. Observa-se menor prevalência nos últimos 4 anos.

Os artigos selecionados contemplam o cenário mundial, sendo prevalentes no Brasil (05 - 33,3\%), nos Estados Unidos (03-20\%), 2 (13,3\%) na Jordânia (02 -13,3\%), na China, Grã-Bretanha, Austrália, Cuba e Gana, respectivamente (01 - 6,6\%).

A pesquisa possibilitou identificar a produção de conhecimento sobre as boas práticas no uso de SE em pacientes críticos em periódicos nacionais e internacionais. Observou-se que a ocorrência de mais estudos quantitativos, como por exemplo do tipo transversal, exploratório, prospectivo e tricêntrico, com diferentes abordagens metodológicas, conforme pode ser verificado no Quadro 1. 
Quadro 1 - Quadro de categorização dos artigos quanto título, autores e periódico. Rio de Janeiro, RJ, Brasil, 2019 (N = 15).

\begin{tabular}{|c|c|c|c|}
\hline $\mathbf{N}^{\mathbf{o}}$ & Título & Autores & Periódico \\
\hline 01 & Cortrak superuser competency assessment and training recommendat & Bourgault, et al & $\begin{array}{c}\text { Journal of Critical } \\
\text { Care }\end{array}$ \\
\hline 02 & $\begin{array}{c}\text { Adherence to standard nursing protocols on nasogastric tube feeding in } \\
\text { asecondary referral hospital in ghana: comparing self-ratings by } \\
\text { professional and auxiliary nurses. }\end{array}$ & Alhassan, et al & $\begin{array}{l}\text { Bmc Health Servics } \\
\text { Research }\end{array}$ \\
\hline 03 & Feeding Tube Securement In Critical Illness: Implications For Safety & Taylor, et al. & $\begin{array}{l}\text { British Journal of } \\
\quad \text { Nursing }\end{array}$ \\
\hline 04 & $\begin{array}{l}\text { Improved safety and efficacy of small-bore feeding tube confirmation } \\
\text { using an electromagnetic placement device }\end{array}$ & Powers, et al & $\begin{array}{l}\text { Nutrition In Clinical } \\
\text { Practice }\end{array}$ \\
\hline 05 & $\begin{array}{c}\text { Blind bedside postpyloric placement of spiral tube as rescue therapy in } \\
\text { Critically ill patients: a prospective, tricentric, observational study }\end{array}$ & lv, et al & Critical care \\
\hline 06 & $\begin{array}{l}\text { Intensive care nurses knowledge of enteral nutrition: a descriptive } \\
\text { questionnaire }\end{array}$ & Morphet, et al & $\begin{array}{l}\text { Intensive And Critical } \\
\text { Care Nursing }\end{array}$ \\
\hline 07 & Terapia de nutrição enteral: construção e validação de protocolo & Campos, et al & $\begin{array}{l}\text { Revista de } \\
\text { Enfermagem UERJ }\end{array}$ \\
\hline 08 & Investigating critical care nurses' perception regarding enteral nutrition & Darawad, et al & Nurse education today \\
\hline 09 & Jordanian Critical Care Nurses' Practices Regarding Enteral Nutrition & Hammad, et al & Critical Care Nurse \\
\hline 10 & $\begin{array}{l}\text { Sondagem enteral: concordância entre teste de ausculta e raio-x na } \\
\text { determinação do posicionamento da sonda }\end{array}$ & Beghetto, et al & $\begin{array}{l}\text { Revista Eletrônica de } \\
\text { Enfermagem }\end{array}$ \\
\hline 11 & $\begin{array}{c}\text { Validação de conteúdo de instrumento sobre a habilidade em sondagem } \\
\text { nasogástrica }\end{array}$ & Medeiros, et al. & $\begin{array}{l}\text { Revista Eletrônica de } \\
\text { Enfermagem }\end{array}$ \\
\hline 12 & $\begin{array}{c}\text { Nursing intervention bundle for enteral nutrition in intensive care: } a \\
\text { collective construction }\end{array}$ & Colaço, et al & $\begin{array}{l}\text { Revista da Escola de } \\
\text { Enfermagem da USP }\end{array}$ \\
\hline 13 & $\begin{array}{c}\text { Factors influencing critical care nurses' adoption of the aacn practice } \\
\text { alert on verification of feeding tube placement }\end{array}$ & $\begin{array}{c}\text { Bourgault, A. } \\
\text { M. et al }\end{array}$ & $\begin{array}{l}\text { American } \\
\text { Journal of Critical } \\
\text { Care }\end{array}$ \\
\hline 14 & Adverse events related to the use of enteral nutritional therapy & Cervo, et al & $\begin{array}{l}\text { Revista Gaúcha de } \\
\text { Enfermagem }\end{array}$ \\
\hline 15 & $\begin{array}{l}\text { Nursing care to patients using gastrointestinal tube: integrative review } \\
\text { of the main problems }\end{array}$ & $\begin{array}{l}\text { Da Silva } \\
\text { Medeiros, et al }\end{array}$ & $\begin{array}{c}\text { Revista Cubana de } \\
\text { Enfermería }\end{array}$ \\
\hline
\end{tabular}

Fonte: Dados da pesquisa (2019). 
Após a leitura atenta do conteúdo dos artigos, os temas que emergiram foram agrupados em quatro eixos temáticos segundo análise e perspectivas dos autores, a saber: a) "Uso de protocolos relacionados ao uso de SE"; b) "Verificação do posicionamento de SE"; c) "Conhecimento sobre TNE" e d) "Prevenção de eventos adversos relacionados à SE", descritos a seguir.

O "Uso de protocolos relacionados ao uso de SE" descreve e discute a importância da elaboração, validação e adesão a protocolos institucionais e a importância do envolvimento dos enfermeiros. Evidencia-se a necessidade da implantação de protocolos que norteiem a realização da assistência das pessoas alimentadas por sonda nos serviços de saúde.

Em relação ao eixo temático "Verificação do posicionamento de SE", foram descritas as formas de confirmação da localização do dispositivo, bem como suas vantagens e desvantagens. Quanto ao "Conhecimento sobre TNE", foram agrupados artigos cuja abordagem corresponderam à percepção dos enfermeiros à TNE, aos cuidados de enfermagem correspondentes e as falhas identificadas neste processo. A "Prevenção de eventos adversos relacionados à SE" abrangeu a retirada não planejada da SE e suas consequências.

\section{Protocolos relacionados ao uso de SE}

As barreiras-chave para a adesão aos protocolos padrão na alimentação por sonda são oportunidades limitadas para treinamentos em serviço e insuficiência dos protocolos de alimentação por sonda nas enfermarias. A necessidade de mais treinamentos rotineiros em serviço com vistas à atualização de conhecimentos pela equipe de enfermagem sobre a alimentação por sonda, além da gerência hospitalar disponibilizar protocolos de enfermagem atuais para guiar a prática dos enfermeiros, juntamente com supervisão da implementação do protocolo por esses profissionais (Alhassam et al, 2019).

O enfermeiro possui muitas atribuições no âmbito da nutrição enteral, que contribuem para o sucesso do tratamento do paciente, como a orientação para o paciente e família em relação à TNE, prescrição dos cuidados de enfermagem, registro das informações pertinentes, realização de atividades de treinamento e educação continuada, além de criação e padronização dos procedimentos de enfermagem e protocolos direcionados à nutrição enteral (Medeiros et al, 2014).

Os protocolos mostram-se importantes, visto a possibilidade de eventos adversos relacionados ao uso da SE. O impacto da fixação da SE no freio nasal, em relação à perda deste dispositivo, bem como as principais complicações a partir desse incidente mostram-se relevantes. A perda inadvertida da SE com este tipo de fixação, caiu de 53\% para 9\%. E a não reinserção da SE aumentou de 20\% para 64\% (Taylor et al, 2018).

É imprescindível que a SE permaneça no local correto, para que não haja deslocamento inadvertido, trazendo riscos ao paciente. Para tanto, a adoção de estratégias e técnicas que garantam a fixação segura do dispositivo é necessária (Colaço \& Nascimento, 2014).

A SE deve ser fixada adequadamente para que os riscos de lesão de mucosa nasal e saída inadvertida sejam minimizados. Podem ser utilizados para fixação fitas/ adesivos hipoalergênicos ou outros produtos de baixo custo e mais acessíveis como: esparadrapo, adesivos permeáveis ou transparentes. A sonda é fixada no nariz com a fita mais adequada, com a finalidade de prevenir lesões resultantes de fricção (UNA -SUS, 2014). A padronização por meio de protocolos é considerada uma ferramenta de gerenciamento nos dias atuais, pois é o caminho mais seguro para a produtividade e a competitividade, constituindo uma das bases do gerenciamento moderno (Campos et al, 2016).

Alguns aspectos das práticas de nutrição enteral parecem ser consistentes com as melhores evidências atuais, como o tempo de iniciação da nutrição enteral e a elevação da cabeceira. Entretanto, alguns outros aspectos apresentam variações e inconsistências com as melhores evidências atuais, tais como a quantidade de volume residual gástrico elevado e seu manejo (Hammad, Hussami \& Darawad, 2015). 
Em relação à subalimentação, é cada vez mais comum pacientes hospitalizados não receberem o volume prescrito e necessário para suas carências metabólicas, o que pode agravar o quadro de desnutrição e como consequência queda na resposta imunológica, abrindo portas para possíveis quadros infecciosos, além do aumento de internação, custos hospitalares e morbidade/mortalidade (Ribas, Garcia \& Abib, 2014).

A validação de instrumentos sobre a habilidade em sondagem nasogástrica, que corresponderam aos passos da técnica, por meio de recomendações atualizadas na literatura demonstram confiabilidade e fidedignidade para observar a qualidade da assistência de enfermagem e pode ser utilizado por serviços de saúde e instituições formadoras para avaliar a habilidade dos profissionais e alunos de graduação (Medeiros et al, 2015; Colaço \& Nascimento, 2014). São exemplos: a pHmetria para confirmação do posicionamento da sonda à beira leito, fixação da sonda, posicionamento entérico da sonda; e manutenção da cabeceira elevada a $30^{\circ}-45^{\circ}$ (Colaço et al, 2014).

Ficou evidenciado que essas intervenções eleitas não demandam acréscimo à carga de trabalho dos profissionais, tampouco encargos financeiros extras à instituição, o que se aponta como potencializadoras na adoção de bundle pelos profissionais de enfermagem (Colaço \& Nascimento, 2014).

Outros fatores que influenciam a prática de verificação da SE são: o uso de uma variedade de métodos à beira do leito para prever localização do tubo durante o procedimento de inserção; o reconhecimento de que os métodos auscultatórios (bolus de ar) não são confiáveis; a obtenção da confirmação radiográfica de correta colocação de qualquer tubo inserido às cegas antes do uso inicial para alimentação ou administração de medicamentos e a verificação da localização do tubo em intervalos de 4 horas após as alimentações são iniciadas (Bourgault et al , 2014).

\section{Verificação do posicionamento de SE}

A inserção de sonda de alimentação assistida por dispositivo de posicionamento eletromagnético, como o Sistema de Acesso Enteral CORTRAK - procedimento em que a sonda enteral (de localização pós-pilórica) é introduzida utilizando um guia magnético e conta com as tecnologias de endoscopia e fluoroscopia - permite que os enfermeiros interpretem a colocação do cateter em tempo real (Borgoult et al, 2019).

A segurança e eficácia do uso de Dispositivo de Posicionamento Eletromagnético (EMPD) pós pilóricos, mostra-se relevante visto que pode minimizar as complicações pulmonares, aumentar a colocação bem-sucedida e diminuir a exposição à radiação do paciente (Powers et al, 2018).

A confirmação radiográfica foi utilizada em 0\% -29,2\% dos tubos colocados em EMPD, e a colocação bem-sucedida de tubos de alimentação usando EMPD variou de $94 \%$-99,6\%. Durante um período de 5 anos, 7.081 tubos de alimentação EMPD foram avaliados. Um evento adverso, pneumotórax, ocorreu durante a colocação desses tubos, correspondendo a uma taxa de $0,014 \%$ (Powers et al, 2018).

Em relação à concordância entre o teste ausculta e a radiografia abdominal na detecção do posicionamento da sonda enteral, a radiografia mostrou $70 \%$ das sondas enterais no estômago, $27,4 \%$ no duodeno, $1,3 \%$ no esôfago e $1,3 \%$ no pulmão direito. O teste de ausculta não detectou duas condições de risco: sonda enteral no esôfago e no brônquio (Beghetto et al, 2015).

Intercorrências relacionadas ao cateter gastrointestinal ainda estão presentes, como posicionamento inadequado no cérebro, trato respiratório e esôfago. Ressalta-se que podem ocorrer equívocos em pacientes com nível de consciência diminuído, tosse e/ou reflexo de vômito suprimidos (Colaço \& Nascimento, 2014).

Segundo os autores, após a confirmação do posicionamento da SE, na medida em que forem administrados alimentos e medicamentos, o tubo deverá ser verificado em outras ocasiões, para que se tenha certeza de que está corretamente 
posicionado. Através de técnicas indicadas como: medição do comprimento da parte exposta do tubo, avaliação visual da coloração e volume do aspirado, medição do ph do aspirado e ausculta do ar (Colaço \& Nascimento, 2014).

A segurança e a eficiência da colocação pós-pilórica cega à beira do leito de um tubo espiral como terapia de resgate, após a falha da migração transpilórica espontânea em pacientes criticamente enfermos mostra-se importante. Em 81,9\% dos pacientes, os tubos de alimentação em espiral foram colocados pós-píloricamente; destes, 55,1\% foram colocados na terceira porção do duodeno ou mais, e 33,9\% foram colocados no jejuno proximal, com um número médio de tentativas de 1,4. O comprimento médio de inserção foi de $95,6 \mathrm{~cm}$. A incidência de eventos adversos foi de $26,0 \%$, e nenhum evento adverso grave foi observado (Lv Bo, 2017).

\section{Conhecimento acerca da TNE}

O conhecimento de enfermeiros sobre a TNE relaciona-se à sua prática de cuidados em relação a esse dispositivo. No entanto, muitos parecem não saber sobre o efeito da desnutrição nos desfechos dos pacientes (Morphet, Clarke \& Bloomer, 2016).

Os cuidados de enfermagem são indispensáveis e contribuem significativamente para o sucesso da TNE. Entre eles, predominam o monitoramento do paciente e manutenção da função do tubo, fornecimento de higiene oral e nasal, monitoramento e manejo de complicações potenciais, redução do risco de aspiração, registro do volume de soluções, alimentações e irrigação (Hinkle \& Cheever, 2016).

Enfermeiros em hospitais privados marcaram a mais alta pontuação em relação à responsabilidade e apoio da documentação. Educação, internet e escola de enfermagem foram as principais fontes de conhecimento sobre nutrição enteral. Além disso, as enfermeiras do sexo feminino diferiram significativamente dos enfermeiros do sexo masculino em relação à percepção de responsabilidade da nutrição enteral (Darawada et al, 2015).

As principais falhas relacionadas ao conhecimento e prática de cuidados de enfermagem aos pacientes submetidos ao uso do trato gastrintestinal referem-se à assistência de Enfermagem em pacientes alimentados por sonda, que contemplam além da técnica, o conhecimento acerca da alimentação enteral (Medeiros et al, 2014).

Os estudos mostraram falhas na assistência de enfermagem por limitações teóricas e/ou por negligência em determinadas situações, com divergências entre o que é praticado e o que é preconizado nas diretrizes da NE.

As principais falhas concentraram-se no conhecimento e prática quanto a aspectos que devem ser avaliados antes da administração da dieta, como verificação de estase gástrica e teste para certificação do posicionamento da sonda, além das condições que contraindicam a nutrição enteral e os riscos e benefícios do uso de sonda para alimentação.

Foram observadas também falhas na comunicação e nos registros de enfermagem, bem como na assepsia durante o manejo da nutrição enteral. Quanto aos sinais de complicação nos pacientes alimentados por sonda, foi verificado a falta de conhecimento em relação à complicação mais grave e a abordagem do paciente nessas situações.

\section{Prevenção de eventos adversos relacionados à SE}

A ocorrência de eventos adversos relacionados ao uso de nutrição enteral se dá através da utilização de indicadores de qualidade que podem estar relacionados à saída inadvertida, obstrução e volume de dieta infundida (Cervo et al, 2014).

A saída inadvertida do tubo e a obstrução podem apresentar taxas de incidência variáveis de acordo com os serviços. O volume de dieta infundido menor que o prescrito, deve-se a pausas para higiene corporal, exames e procedimentos, náuseas / vômitos. Para prevenção de tais eventos, a utilização de protocolos institucionais direcionados são relevantes e sua adesão deve ser estimulada pelos gestores. 
Esse foi o único estudo encontrado que traz a discussão acerca os eventos adversos que podem ocorrer no dia a dia das equipes na UTI em relação à SE, levando em consideração que outras equipes multidisciplinares além da enfermagem também podem estar relacionadas à prevenção de incidentes que envolvem a SE.

\section{Conclusão}

Observou-se que os artigos chamam atenção para as responsabilidades do enfermeiro no cuidado ao paciente com SE, como a orientação para o paciente e família em relação à TNE, além da prescrição de cuidados de enfermagem, registro das informações pertinentes, realização de atividades de treinamento/atualizações em equipe bem como a elaboração e supervisão de protocolos direcionados à nutrição enteral.

Verificou-se que a boas práticas referentes ao uso de SE se concentraram no conhecimento e prática de aspectos como verificação de estase gástrica e teste para certificação do posicionamento da sonda, além das condições que contraindicam a nutrição enteral. Além disso, observou-se a importância das orientações à família e equipes e principalmente cumprimento dos protocolos institucionais.

Espera-se que os dados resultantes desse estudo possam contribuir para a melhoria da assistência ao paciente em uso de SE, tendo em vista a importância desse dispositivo para o tratamento e promoção da saúde do paciente na UTI. Sugere-se a investigação das boas práticas relacionadas ao uso de SE em diferentes contextos de saúde.

\section{Referências}

Alhassan, R. K., et al. (2019). Adherence to standard nursing protocols on nasogastric tube feeding in a secondary referral hospital in Ghana: comparing selfratings by professional and auxiliary nurses. BMC Health Services Research, 19(1), 119-27. http://dx.doi.org/10.1186/s12913-019-3931-6

Beghetto, M. G., Anziliero, F., Leães, D. M., \& Mello, E. D de. (2015). Feeding tube placement: auscultatory method and x-ray agreement. Revista Gaúcha de Enfermagem, 36(4), 98-103. http://dx.doi.org/10.1590/1983-1447.2015.04.54700

Botelho, L. L. R., Cunha, C. C. de A., \& Macedo M. (2011). The integrative review method in organizational studies. Rev Eletr Gestão Soc, 5(11), 121-136. https://www.gestaoesociedade.org/gestaoesociedade/article/view/1220

Bourgault, A. M., Gonzalez, L., Aguirre, L., \& Ibrahim, J. A. (2019). CORTRAK Superuser Competency Assessment and Training Recommendations. American Journal Of Critical Care, 28(1), 30-40. http://ajcc.aacnjournals.org/content/28/1/30.long

Bourgault, A. M. et al. (2014). Factors Influencing Critical Care Nurses' Adoption of the AACN Practice Alert on Verification of Feeding Tube Place ment. American Journal Of Critical Care, 23(2), 134-144. http://dx.doi.org/10.4037/ajcc2014558

Campos, F. de A., Caetano, J. A., Almeida, P. C. de, \& Da Silva, V. M. (2016). Terapia de nutrição enteral: construção e validação de protocolo Enteral nutrition therapy. Revista Enfermagem UERJ, 24(2), 1-5. http://dx.doi.org/10.12957/reuerj.2016.11625

Cervo, A. S. et al. (2014). Adverse events related to the use of enteral nutritional therapy. Revista Gaúcha de Enfermagem, 35(2), 53-59. http://dx.doi.org/10.1590/1983-1447.2014.02.42396

Colaço, A. D., \& Nascimento, E. R. P do. (2014). Nursing intervention bundle for enteral nutrition in intensive care: a collective construction. Revista Escola de Enfermagem da USP, 48(5), 841-847. https://pdfs.semanticscholar.org/1563/06375248efc704549d0a57d67f1ec939e5da.pdf

Darawad, M. W. et al. (2015). Investigating critical care nurses' perception regarding enteral nutrition. Nurse Education Today, 35(2), 414-419. http://dx.doi.org/10.1016/j.nedt.2014.11.023

De Souza, I. A. et al. (2018). Nutrição enteral em pacientes oncológicos: diferenças entre o que é prescrito e administrado. Nutricion Clinica y Dietetica Hospitalaria, 38(2), 31-38. http://dx.doi.org/10.12873/382Iury

Hammad, S. M., Al-Hussami, M., \& Darawad, M. W. (2015). Jordanian Critical Care Nurses’ Practices Regarding Enteral Nutrition. Gastroenterology Nursing, 38(4), 279-288. http://dx.doi.org/10.1097/sga.0000000000000133

Hinkle, J. L., \& Cheever, K. H. (2016). Brunner e Suddarth: Tratado de Enfermagem Médico-Cirúrgica (13a ed). Editora Guanabara Koogan.

Lv, B. et al. (2017). Blind bedside postpyloric placement of spiral tube as rescue therapy in critically ill patients: a prospective, tricentric, observational study. Critical Care, 21(1), 248-255. http://dx.doi.org/10.1186/s13054-017-1839-2

Medeiros, R. K. da S. et al (2014). Assistência de enfermagem a pacientes em uso de sonda gastrointestinal: revisão integrativa das principais falhas. Revista Cubana de Enfermería, 30(4). http://www.revenfermeria.sld.cu/index.php/enf/article/view/288 
Research, Society and Development, v. 10, n. 4, e53410414468, 2021

(CC BY 4.0) | ISSN 2525-3409 | DOI: http://dx.doi.org/10.33448/rsd-v10i4.14468

Medeiros, R. K. da S. et al. (2015). Validação de conteúdo de instrumento sobre a habilidade em sondagem nasogástrica. Revista Eletrônica de Enfermagem, 17(2), 278-289. http://dx.doi.org/10.5216/ree.v17i2.28820

Morphet, J., Clarke, A. B., \& Bloomer, M. J. (2016). Intensive care nurses' knowledge of enteral nutrition: A descriptive questionnaire. Intensive And Critical Care Nursing, 37, 68-74. http://dx.doi.org/10.1016/j.iccn.2016.07.001

Nettina, S. M. (2014). Prática de Enfermagem (8a ed.). Editora Guanabara Koogan.

O'Leary-Kelley, C., \& Bawel-Brinkley, K. (2017). Nutrition Support Protocols: Enhancing Delivery of Enteral Nutrition. Crit Care Nurse, 37(2), e15-e23. $10.4037 / \operatorname{ccn} 2017650$

Pereira, L. M. V. et al. (2018). Retirada não planejada de dispositivos invasivos e suas implicações para a segurança do paciente crítico. Revista de Pesquisa: Cuidado é Fundamental Online, 10(2), 490-495. http://www.seer.unirio.br/index.php/cuidadofundamental/article/view/6106

Powers, J. et al. (2018). Improved Safety and Efficacy of Small-Bore Feeding Tube Confirmation Using an Electromagnetic Placement Device. Nutrition In Clinical Practice, 33(2), 268-273. http://dx.doi.org/10.1002/ncp.10062

Ramalho Neto, J. M., Marques, D. K. A., Fernandes, M. G. M., \& Nóbrega, M. M. L da. (2016). Análise de teorias de enfermagem de Meleis: revisão integrativa. Revista Brasileira de Enfermagem, 69(1), 174-181. https://doi.org/10.1590/0034-7167.2016690123i

Ribas, B. L. P., Garcia, R. S., \& Abib, R. T. (2014). Motivos para interrupção da terapia nutricional enteral em pacientes hospitalizados. Revista Brasileira Nutrição Clinica, 29(4), 331-334. http://www.braspen.com.br/home/wp-content/uploads/2016/11/10-Motivos-para-interrup\%C3\%A7\%C3\%A3o-daterapia.pdf

Taylor, S. J., Allan, K., Clemente, R., Marsh, A., \& Toher, D. (2018). Feeding tube securement in critical illness: implications for safety. British Journal Of Nursing, 27(18), 1036-1041. http://dx.doi.org/10.12968/bjon.2018.27.18.1036

UNA-SUS/UFPE, Universidade Federal de Pernambuco. (2014). Fixação da sonda nasoenteral.

Viana, R. A. P. P., \& Torre, M. (2016). Enfermagem Em Terapia Intensiva-Práticas Integrativas. Manole. 